\title{
Implementation of nearly single-mode second harmonic generation by using a femtosecond laser written waveguiding structure in $\mathrm{KTiOPO}_{4}$ nonlinear crystal
}

\author{
Weijie Nie ${ }^{\mathrm{a}}$, Carolina Romero ${ }^{\mathrm{b}}$, Qingming $\mathrm{Lu}^{\mathrm{c}}$, Javier Rodríguez Vázquez de Aldana ${ }^{\mathrm{b}}$, \\ Feng Chen ${ }^{\mathrm{a}, *}$ \\ a School of Physics, State Key Laboratory of Crystal Materials, Shandong University, Jinan, 250100, China \\ b Laser Microprocessing Group, Facultad Ciencias, Universidad de Salamanca, Salamanca, 37008, Spain \\ ${ }^{\mathrm{c}}$ School of Chemistry and Chemical Engineering, Shandong University, Jinan, 250100, China
}

A R T I C L E I N F O

\section{Keywords:}

Optical waveguides

Femtosecond-laser writing

Nonlinear optical materials

\begin{abstract}
A B S T R A C T
We propose a hybrid photonic structure to realize guided-wave second harmonic generation (SHG) from nearinfrared (NIR at $1064 \mathrm{~nm}$ ) to visible (green light at $532 \mathrm{~nm}$ ) wavelength with nearly single-mode output. The periodically arrayed tracks have been produced in $\mathrm{KTiOPO}_{4}$ nonlinear crystal by femtosecond laser writing, which enable efficient light field confinement with cladding-like refractive index distributions. Particularly, the track-cladding surrounded central region is free of any tracks, which serves as waveguiding cores. With designed core diameters, the structures could enable single-mode propagation at selected wavelength regime on purpose. In this work, the hybrid structure contains a larger-input core section and a connected smaller-output core section, which in principle supports nearly single mode for either fundamental pump beam or second harmonic beam in the input and output channel, respectively. Based on this hybrid structure we implement nearly singlemode SHG at $532 \mathrm{~nm}$, and comparable normalized conversion efficiency $(1.1 \% / \mathrm{W} / \mathrm{cm})$ in the continuous-wave (CW) regime is obtained with respect to that $(1.2 \% / \mathrm{W} / \mathrm{cm})$ of multimode SHG from a single large-core channel structure. This work paves the way to realize mode profile controlling for selected wavelength by using laserwritten arrayed tracks.
\end{abstract}

\section{Introduction}

Optical waveguides are basic components in integrated photonic systems, in which light propagation could be confined and tailored in channels with diffraction-free geometries [1,2]. In addition, owing to the compact configurations of waveguide-based devices, numerous optical applications have been realized in various waveguide systems. For example, waveguide amplifiers and electro-optical modulators have been widely applied into the telecommunication systems $[3,4]$. Waveguide lasers could be utilized as miniature light sources [5-7]. Waveguide arrays and lattices have potential applications in quantum photonics to realize on-chip computing [8], memory storage [9], and other intricate information processing $[10,11]$. In integrated nonlinear optics, the mode control of light at diverse wavelengths may be a technical topic for practical applications. For SHG in normal waveguides, the fundamental and the second harmonic (SH) light may not be simultaneously at same order mode because the criteria for fundamental and
SH waves are different for a given order [12]. In practice, single-mode light is often required due to the high efficiency of the nonlinear optical process and easy manipulation of beam profiles. To achieve singlemode SHG in nonlinear waveguides, one may use specially designed guiding structures.

Femtosecond laser writing has become a powerful technique to fabricate optical waveguides in transparent optical materials [13-16], since in 1996 Davis et al. reported on the first femtosecond-laser written waveguides in a few family glasses [17]. Compared with other techniques for waveguide fabrication, direct femtosecond laser writing possesses unique three-dimensional (3D) processing capability of materials, which enables implementation of devices with various functionalities in dielectrics $[18,19]$. As a result, highly efficient miniaturized platforms based on laser-written waveguides have been realized for photonic signal processing towards diverse purposes [20-25]. The laser processing of single crystals is much more complicated than glasses due to the versatile crystalline structures and diverse physical

\footnotetext{
* Corresponding author.

E-mail address: drfchen@sdu.edu.cn (F. Chen).
} 
properties of crystals. The so-called Type I and II modification usually refers positive and negative refractive index changes inside the laserinduced tracks, respectively [26]. The advantage of Type I modification is the easy fabrication of 3D devices; however, it has been only realized in a few crystals, such as $\mathrm{LiNbO}_{3}[27,28]$ and BGO [29], and may be also with polarization-sensitive guidance. For most crystals, Type II modification is more common and easy to achieve, but the 3D engineering of the waveguide is relatively difficult to realize by directly using negative index changed tracks. Nevertheless, several 2D waveguide structures, such as beam splitters and interferometers, have been produced based on the well-known Type II double-line approach [30-32]. Like Type II modification with reduced refractive index, depressed cladding waveguides (also called Type III waveguides), located in the region surrounded by these negative-index-changed tracks, have superior performance in development of 3D devices. These novel structures are with polarization-independent guidance on the cross section and preserved bulk features in the waveguide core region, which are beneficial to applications such as waveguide lasing, SHG, and light amplification [26,33]. Based on these advantages, recently we have proposed and fabricated numerous promising guiding structures with hexagonal geometry of periodically-arrayed tracks (so-called optical-lattice-like structures) to implement 3D beam splitting and ringshaped transformation of beam profiles [34]. Furthermore, the 3D lasing and SHG have also been realized by using specially designed hybrid structures (i.e., combination of a few different track lattice structures) [35]. By introducing new axial defects in the appropriate positions of the lattice, diverse multi-functional microstructures could be designed combining the original crystal properties. In this work, we propose a two-element hybrid structure to achieve mode controlling in nonlinear KTP crystal, which is low-cost compared to PPKTP based on quasi-phase matching [36,37]. The hybrid structure consists of two connected optical-lattice-like structures with different guiding core diameters, which are used for nearly single-mode confinement of input fundamental and output SH waves, respectively. With this hybrid structure we experimentally realized SHG of green light at $532 \mathrm{~nm}$. For comparison, two single structures corresponding to two elements of the hybrid structure have been employed with the implementation of double frequency at the wavelength of $532 \mathrm{~nm}$, respectively.

\section{Experimental}

The hybrid structure and the compared single structures are fabricated by femtosecond laser writing in a $\mathrm{KTiOPO}_{4}$ crystal with the dimension of $10 \times 5 \times 2 \mathrm{~mm}^{3}$. After optically polished, the crystal is inscribed along $10 \mathrm{~mm}$ orientation for Type II $\left(\mathrm{e}^{\omega}+\mathrm{o}^{\omega} \rightarrow \mathrm{e}^{2 \omega}\right)$ phase matching (PM) SHG (i.e., $\theta=90^{\circ}, \phi=23.5^{\circ}$ ). The fabrication process is realized by using the laser facility in the Universidad de Salamanca, Spain. The laser source which is delivered by an amplified Ti:Sapphire femtosecond-laser (Spitfire, Spectra Physics) generates linearly-polarized pulses with 120 -fs temporal duration and $1-\mathrm{kHz}$ repetition rate at a central wavelength of $800 \mathrm{~nm}$. The measured pulse energy of $8 \mu \mathrm{J}$ is reduced by a calibrated neutral density filter placed after a set of halfwave plate and a linear-polarization cube. After converged by a $40 \times$ microscope objective, the pulse has the energy of $0.17 \mu \mathrm{J}$ to scan at constant velocity of $650 \mu \mathrm{m} / \mathrm{s}$ beneath one of sample surface $\left(10 \times 5 \mathrm{~mm}^{2}\right)$, producing a negative refractive-index track with transverse length of $\sim 10 \mu \mathrm{m}$ inside the crystal. The sample is located on a computer-controlled 3D-motorized stage and this scanning process is repeated at different positions of the sample, following the desired hexagonal geometry with a lateral separation of $10 \mu \mathrm{m}$ between each two adjacent damage tracks, resulting in the optical-lattice-cladding microstructures formation. By introducing the damage line in appropriate positions, the microstructures with different core diameters can be obtained to steer the beam propagation. As shown in Fig. 1, the hybrid structure (hereafter by HS) is combined by a $5.5-\mathrm{mm}$ single structure with large core (hereafter by SSL) as input element and a 4.5-

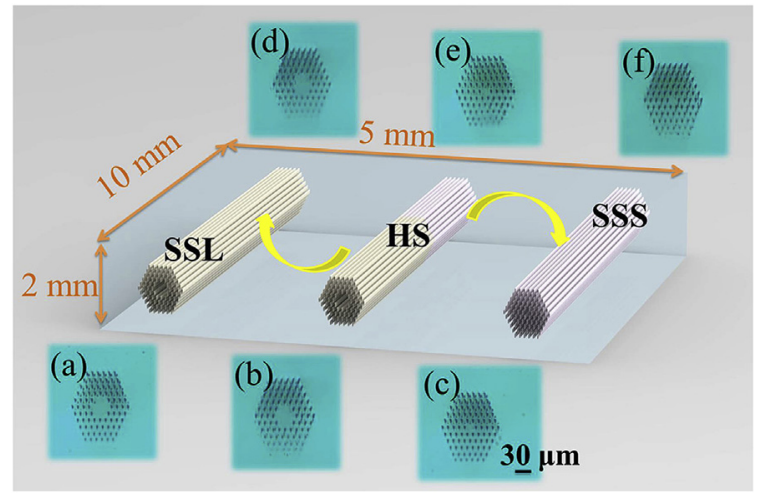

Fig. 1. The structure plots of optical-lattice-like cladding waveguides Nos. 1-3 in details. (a)-(c) is the cross-sectional microscope images of input face in optical-lattice-like cladding waveguides Nos.1-3, and (d)-(f) is the cross-sectional microscope images of output face in optical-lattice-like cladding waveguides Nos. 1-3, respectively.

mm single structure with small core (hereafter by SSS) as output element. The inset of Fig. 1 exhibits all the input (Fig. 1(a)-(c)) and output (Fig. 1(d)-(f)) microscope images of SSL, HS and SSS, respectively. Due to the combination, the input microscope image of HS (Fig. 1(b)) is same with SSL (Fig. 1(a)), whilst the output one of HS (Fig. 1(e)) is same with SSS (Fig. 1(f)). For comparison, an SSL and an SSS with length of $10 \mathrm{~mm}$ (i.e., with same length to the HS) are also produced by laser writing.

The attenuations of all the waveguide microstructures are estimated by directly measuring the input and output power of the transmitted light based on a typical end-face coupling arrangement. Considering the mismatch coefficient of $0.75,0.75$ and 0.88 between the launched Gaussian field profiles and the modal profiles of the microstructure simulated by FD-BPM algorithm (Rsoft ${ }^{\circledR}$ Beam PROP) [38] for SSL, HS and SSS, the propagation losses are determined to be $1.2,1.6,1.5 \mathrm{~dB} /$ $\mathrm{cm}$ along TM polarization and $1.4,1.6,1.4 \mathrm{~dB} / \mathrm{cm}$ along TE polarization at $633 \mathrm{~nm}$, respectively, which is described in Table 1 . The tiny additional losses of HS could be partly attributed to the imperfections of two elements. It should be noted that the propagation losses for all the waveguides is almost same for TE and TM mode, which is beneficial to generate high efficient SH laser with Type II PM.

SHG nonlinear experiments are implemented through a similar endface coupling system in the continuous-wave (CW) regime, as demonstrated in Fig. 2. A linearly-polarized laser at $1064 \mathrm{~nm}$ is applied as the fundamental incident source. To mix equal "e" and "o" components of the fundamental laser for satisfying the $1 / 2 \mathrm{e}^{\omega}+1 / 2^{\circ \omega}$ of Type II PM SHG, a half-wave plate is used to control the laser polarization along the $45^{\circ}$ direction. After that, the laser is focused by a $20 \times$ microscope objective with N.A. of 0.4 and coupled into the microstructures end-face $\left(5 \times 2 \mathrm{~mm}^{2}\right)$. Then the frequency doubling laser generated from the waveguide is collected by another same objective. Through an opticallow-pass-filter (OLPF) with high transmission of $\sim 90 \%$ at 532-nm and reflectivity $>99 \%$ at $1064 \mathrm{~nm}$, the nonlinear performance can be

Table 1

The nonlinear waveguide properties.

\begin{tabular}{lllll}
\hline Waveguide & $\begin{array}{l}\text { Loss at } \\
633 \mathrm{~nm}(\mathrm{~dB} / \mathrm{cm})\end{array}$ & \multicolumn{2}{l}{ SHG Properties in CW regime } \\
\cline { 2 - 5 } & TE & TM & $\begin{array}{l}\text { Maximum output } \\
\text { power at } \\
532 \mathrm{~nm}(\mathrm{~mW})\end{array}$ & $\begin{array}{l}\text { Normalized Conversion } \\
\text { Efficiency from } 1064 \text { to } \\
532 \mathrm{~nm}(\% / \mathrm{W} / \mathrm{cm})\end{array}$ \\
\hline SSL & 1.2 & 1.4 & 0.84 & 1.2 \\
HS & 1.6 & 1.6 & 0.80 & 1.1 \\
SSS & 1.5 & 1.4 & 0.39 & 0.6 \\
\hline
\end{tabular}




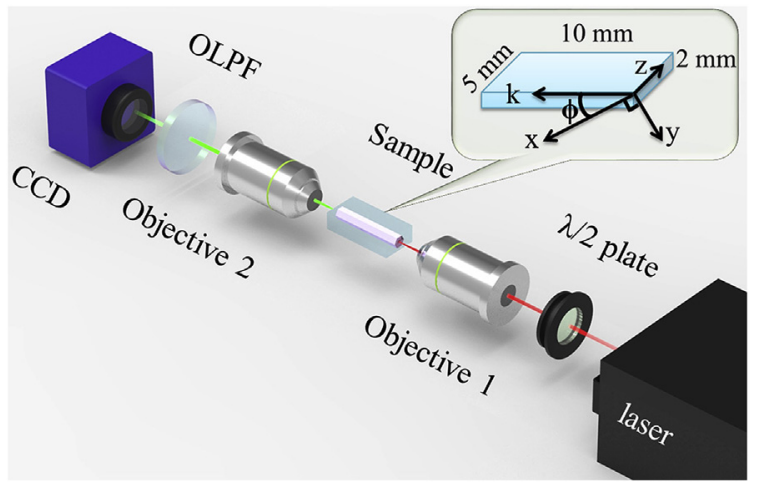

Fig. 2. Schematic of experimental arrangement for second-harmonic-generation in KTP optical-lattice-like cladding waveguides.

characterized by the CCD camera, power-meter, and a spectrometer with resolution of $0.2 \mathrm{~nm}$.

\section{Results and discussion}

As to obtain single-mode fundamental and SH lasers by the waveguide microstructures, a group of optical-lattice-like cladding waveguides is designed, finding an approximate microstructure to be HS for nonlinear performance. During this designing, Rsoft ${ }^{\odot}$ software [38], based on finite-difference beam propagation method (FD-BPM), is utilized for simulating the light modal profiles through various microstructures at different wavelengths. The refractive index change of scanning damage track in the crystal is determined to be -0.0035 , which is similar with our previous work [35]. With this value, the spatial distribution of refractive index could be constructed for SSL and SSS, respectively, as depicted in Fig. 3(a) and (c). Corresponding theoretical laser modal profiles of SSL at $1064 \mathrm{~nm}$ and SSS at $532 \mathrm{~nm}$ are calculated, shown in Fig. 3(b) and (d), respectively, indicating the nearly single modes realization of fundamental laser $(1064 \mathrm{~nm})$ in SSL and frequency doubling laser (532 nm) in SSS. Under this condition, HS could be expected to achieve the simultaneous nearly single-mode output from $1064 \mathrm{~nm}$ to $532 \mathrm{~nm}$ due to the connection of 5.5-mm SSL and $4.5-\mathrm{mm}$ SSS in the sequence.

Fig. 4 shows the measured SH (Fig. 4(a)-(c)) and fundamental (Fig. 4(d)-(f)) near-field intensity distributions through all the

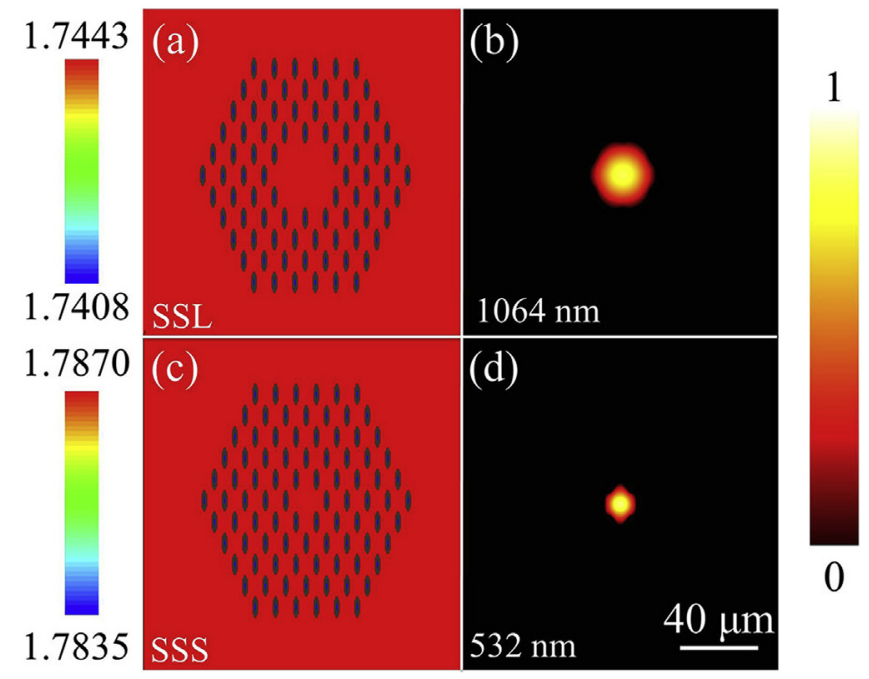

Fig. 3. 2D refractive index profile at the cross section in the waveguides Nos. 1 (a) and 3 (c), respectively. The corresponding calculated modal profile of waveguides Nos.1 at $1064 \mathrm{~nm}$ (b) and 3 at $532 \mathrm{~nm}$ (d), respectively. The scale bar is shown in (d).
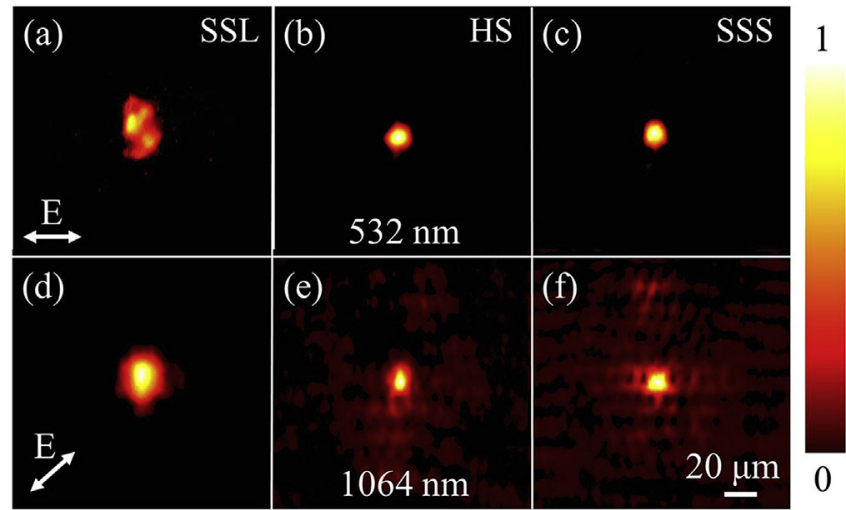

Fig. 4. Near-field modal profiles of optical-lattice-like cladding waveguides Nos. $1-3$ at (a)-(c) $532 \mathrm{~nm}$ and (d)-(f) $1064 \mathrm{~nm}$ under $1064 \rightarrow 532 \mathrm{~nm}$ green laser SHG configuration. The scale bar is shown in (f). (For interpretation of the references to colour in this figure legend, the reader is referred to the Web version of this article.)

microstructures of SSL, HS and SSS, respectively. The arrows in the figure represent the polarization directions of transmitted NIR (Fig. 4(d)) and generated green (Fig. 4(a)) lasers, respectively, which is consistent with the $1064 \rightarrow 532 \mathrm{~nm}$ conversion process of Type II $\left(\mathrm{e}^{\omega}+\mathrm{o}^{\omega} \rightarrow \mathrm{e}^{2 \omega}\right)$ SHG in the $\mathrm{KTiOPO}_{4}$ waveguides. It could be observed that HS waveguide supports well-confined near-single mode of 532-nm laser generation (Fig. 4(b)) and slightly leaky mode of 1064-nm light propagation (Fig. 4(e)). While for the compared waveguides, the multimode laser is delivered from the SSL waveguide at $532 \mathrm{~nm}$ (Fig. 4(a)) due to the large scale of the core and much-leakier-mode fundamental laser compared to the SH mode is transmitted through the SSS waveguide (Fig. 4(f)) because the beam could not be limited in the microstructure at the wavelength of $1064 \mathrm{~nm}$. In addition, the SSL and SSS waveguides display nearly single modes at the wavelengths of $1064 \mathrm{~nm}$ and $532 \mathrm{~nm}$, which are in good agreement with the calculated modal profiles, respectively.

To further investigate the nonlinear properties of all the microstructures in $\mathrm{KTiOPO}_{4}$ crystal, $\mathrm{SH}$ output powers and conversion efficiencies as a function of the 1064-nm input powers are depicted in Fig. 5(a)-(c) from SSL, HS and SSS waveguides, respectively. The experimental solid spots are fitted to be the solid lines by the curve functions, respectively. Briefly, corresponding normalized laser spectra are manifested in Fig. 5(d) with the fundamental waves at $1064 \mathrm{~nm}$ and SH waves at $532 \mathrm{~nm}$, respectively. All the waveguides details about maximum output powers and normalized conversion efficiencies in CW regime are listed in Table 1 . The normalized conversion efficiency is given by the equation

$\eta=P_{2 \omega} /\left(L \cdot\left(P_{\omega}^{i n}\right)^{2}\right)$

Where $P_{2 \omega}$ is the output power of SH laser at $532 \mathrm{~nm}$ and $P_{\omega}{ }^{i n}$ is the input power of fundamental laser at $1064 \mathrm{~nm}$, respectively. $L$ denotes the guided length for SHG. Based on the identical launched power of $263.68 \mathrm{~mW}$ at $1064 \mathrm{~nm}$, the HS waveguide has the 532-nm green laser power of $0.80 \mathrm{~mW}$ which is more than twice higher than similar singlemode output power of $0.39 \mathrm{~mW}$ in SSS waveguide and slightly lower than multi-mode 532-nm laser power of $0.84 \mathrm{~mW}$ from SSL waveguide. Moreover, the normalized conversion efficiency of $1.1 \% / \mathrm{W} / \mathrm{cm}$ from HS is comparable with respect to that of $1.2 \% / \mathrm{W} / \mathrm{cm}$ in SSL waveguide, but nearly twice as much as that of $0.6 \% / \mathrm{W} / \mathrm{cm}$ from SSS waveguide.

It is reasonable for HS waveguide with large-area incident region and small-area output volume that more pumping fundamental power goes into the microstructure, providing more spatial overlap of 1064 and 532-nm light field. Note that the tiny additional loss of HS is another crucial factor that leads to the decrease of 532-nm output power and normalized conversion efficiency when compared with the SSL 

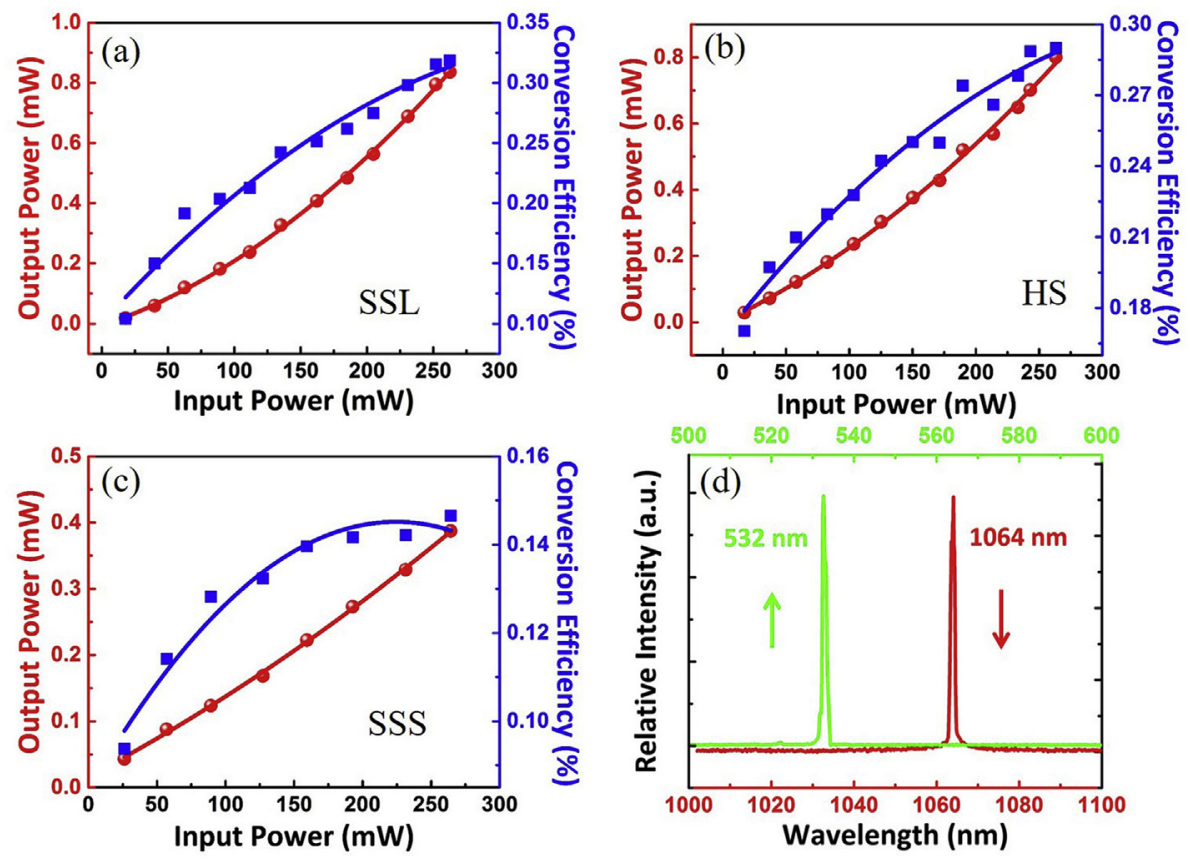

Fig. 5. The measured SHG output power and conversion efficiency as function of the fundamental pump power of (a)-(c) optical-lattice-like cladding waveguides Nos.1-3, respectively. (d) The spectra of the fundamental laser beam at $1064 \mathrm{~nm}$ and the SHG at $532 \mathrm{~nm}$ transmitted through femtosecond-laser inscribed KTP optical-lattice-like cladding waveguides. waveguide. Nonetheless, it is still superior to some reported nonlinear waveguides [35,39]. For example, with similar optical-lattice-like cladding microstructures in $\mathrm{CW}$ regime, the SH laser power of HS ( $0.80 \mathrm{~mW}$ from $263.68-\mathrm{mW}$ launched power) is enhanced with respect to the reported waveguide $(0.68 \mathrm{~mW}$ from $613.3-\mathrm{mW}$ incident power) [35]. Therefore, the HS waveguide implements near-single mode control with relative enhanced SH output power and comparable normalized conversion efficiency from 1064 to $532 \mathrm{~nm}$.

\section{Conclusions}

We demonstrate the specially designed optical-lattice-like hybrid cladding waveguides in a $\mathrm{KTiOPO}_{4}$ crystal to realize nearly single-mode SHG by utilizing femtosecond laser writing. By introducing the damage tracks in designed position, the 10-mm long HS waveguide has been achieved with the integration of 5.5-mm SSL and 4.5-mm SSS waveguides, which provides a simple way to build compact optical-latticelike cladding microstructures capable of producing efficient beam mode controlling. Based on the Type II PM, frequency doubling at $532 \mathrm{~nm}$ has been realized in the HS waveguide with nearly single-mode profiles, corresponding to the maximum normalized conversion efficiency of $1.1 \% / \mathrm{W} / \mathrm{cm}$ in CW regime. It combines the advantages of SSL and SSS waveguides with high normalized conversion efficiency of $1.2 \% / \mathrm{W} / \mathrm{cm}$ and nearly single mode profiles, simultaneously avoids the disadvantages of multimode distribution for SSL waveguide and low normalized conversion efficiency of $0.6 \% / \mathrm{W} / \mathrm{cm}$ for SSS waveguide, respectively. This work paves a way to realize single mode laser generation at the selected wavelength with superior performance for diverse applications in a single chip.

\section{Acknowledgements}

The work is supported by the National Natural Science Foundation of China (61775120); Junta de Castilla y León (Project SA046U16) and MINECO (FIS2013-44174-P, FIS2015-71933-REDT).

\section{References}

[1] Q. Wu, J.P. Turpin, D.H. Werner, Integrated photonic systems based on transformation optics enabled gradient index devices, Light sci. Appl. 1 (2012) e38.

[2] D.N. Christodoulides, F. Lederer, Y. Silberberg, Discretizing light behavior in linear and nonlinear waveguide lattices, Nature 424 (2003) 817-823.

[3] J. del Hoyo, P. Moreno-Zarate, G. Escalante, J.A. Valles, P. Fernandez, J. Solis, High efficiency waveguide optical amplifiers and lasers via fs-laser induced local modification of the glass composition, J. Lightwave Technol. 35 (2017) 2955-2959.

[4] F. Qiu, H. Sato, A.M. Spring, D. Maeda, M. Ozawa, K. Odoi, I. Aoki, A. Otomo, S. Yokoyama, Ultra-thin silicon/electro-optic polymer hybrid waveguide modulators, Appl. Phys. Lett. 107 (2015) 123302.

[5] T. Calmano, S. Müller, Crystalline waveguide lasers in the visible and near-infrared spectral range, IEEE J. Sel. Top. Quant. Electron. 21 (2015) 401-413.

[6] C. Grivas, C. Corbari, G. Brambilla, P.G. Lagoudakis, Tunable, continuous-wave Ti:sapphire channel waveguide lasers written by femtosecond and picosecond laser pulses, Opt. Lett. 37 (2012) 4630-4632.

[7] N. Pavel, G. Salamu, F. Jipa, M. Zamfirescu, Diode-laser pumping into the emitting level for efficient lasing of depressed cladding waveguides realized in $\mathrm{Nd}: \mathrm{YVO}_{4}$ by the direct femtosecond-laser writing technique, Optic Express 22 (2014) 23057-23065.

[8] T.D. Ladd, F. Jelezko, R. Laflamme, Y. Nakamura, C. Monroe, J.L. O'Brien, Quantum computers, Nature 464 (2010) 45-53.

[9] E. Kuramochi, K. Nozaki, A. Shinya, K. Takeda, T. Sato, S. Matsuo, H. Taniyama, H. Sumikura, M. Notomi, Large-scale integration of wavelength-addressable alloptical memories on a photonic crystal chip, Nat. Photon. 8 (2014) 474-481.

[10] T. Meany, M. Gräfe, R. Heilmann, A. Perez-Leija, S. Gross, M.J. Steel, M.J. Withford, A. Szameit, Laser written circuits for quantum photonics, Laser Photon. Rev. 9 (2015) 363-384.

[11] A. Perez-Leija, R. Keil, A. Kay, H. Moya-Cessa, S. Nolte, L. Kwek, B.M. RodríguezLara, A. Szameit, D.N. Christodoulides, Coherent quantum transport in photonic lattices, Phys. Rev. 87 (2013) 012309.

[12] C. Grivas, Optically pumped planar waveguide lasers, Part I: fundamentals and fabrication techniques, Prog. Quant. Electron. 35 (2011) 159-239.

[13] A. Ferrer, A.R. de la Cruz, D. Puerto, W. Gawelda, J.A. Vallés, M.A. Rebolledo, V. Berdejo, J. Siegel, J. Solis, in situ assessment and minimization of nonlinear propagation effects for femtosecond-laser waveguide writing in dielectrics, J. Opt. Soc. Am. B 27 (2010) 1688-1692.

[14] R. Osellame, G. Cerullo, R. Ramponi, Femtosecond-laser Micromachining: Photonic and Mi-crofluidic Devices in Transparent Materials, Springer, Berlin, 2012.

[15] R.R. Gattass, E. Mazur, Femtosecond laser micromachining in transparent materials, Nat. Photon. 2 (2008) 219-225.

[16] F. Zhang, M. Gecevičius, Q. Chen, H. Zhang, Y. Dai, J. Qiu, Evolution of polarization dependent microstructures induced by high repetition rate femtosecond laser irradiation in glass, Optic Express 24 (2016) 21353-21363.

[17] K.M. Davis, K. Miura, N. Sugimoto, K. Hirao, Writing waveguides in glass with a femtosecond laser, Opt. Lett. 21 (1996) 1729-1731.

[18] J. Martínez, A. Ródenas, T. Fernandez, J.R. Vázquez de Aldana, R.R. Thomson, M. Aguiló, A.K. Kar, J. Solis, F. Díaz, 3D laser-written silica glass step-index highcontrast waveguides for the $3.5 \mu \mathrm{m}$ mid-infrared range, Opt. Lett. 40 (2015) 5818-5821.

[19] S. Nolte, M. Will, J. Burghoff, A. Tuennermann, Femtosecond waveguide writing: a new avenue to three-dimensional integrated optics, Appl. Phys. a 77 (2003) $109-111$.

[20] S. Juodkazis, V. Mizeikis, H. Misawa, Three-dimensional microfabrication of materials by femtosecond lasers for photonics applications, J. Appl. Phys. 106 (2009) 051101.

[21] A. Marcinkevičius, S. Juodkazis, M. Watanabe, M. Miwa, S. Matsuo, H. Misawa, 
J. Nishii, Femtosecond laser-assisted three-dimensional microfabrication in silica, Opt. Lett. 26 (2001) 277-279.

[22] Z. Chaboyer, A. Stokes, J. Downes, M.J. Steel, M.J. Withford, Design and fabrication of reconfigurable laser-written waveguide circuits, Optic Express 25 (2017) 33056-33065.

[23] M. Ams, G.D. Marshall, P. Dekker, J.A. Piper, M.J. Withford, Ultrafast laser written active devices, Laser Photon. Rev. 3 (2009) 535-544.

[24] M. Malinauskas, A. Žukauskas, S. Hasegawa, Y. Hayasaki, V. Mizeikis, R. Buividas, S. Juodkazis, Ultrafast laser processing of materials: from science to industry, Light sci. Appl. 5 (2016) e16133.

[25] R.M. Vazquez, R. Osellame, D. Nolli, C. Dongre, H. van den Vlekkert, R. Ramponi, M. Pollnaub, G. Cerullo, Integration of femtosecond laser written optical waveguides in a lab-on-chip, Lab a Chip 9 (2008) 91-96.

[26] F. Chen, J.R. Vazquez de Aldana, Optical waveguides in crystalline dielectric materials produced by femtosecond laser micromachining, Laser Photon. Rev. 8 (2014) 251-275.

[27] R.R. Thomson, S. Campbell, I.J. Blewett, A.K. Kar, D.T. Reid, Optical waveguide fabrication in z-cut lithium niobate $\left(\mathrm{LiNbO}_{3}\right)$ using femtosecond pulses in the low repetition rate regime, Appl. Phys. Lett. 88 (2006) 111109.

[28] J. Burghoff, H. Hartung, S. Nolte, A. Tünnermann, Structural properties of femtosecond laser-induced modifications in $\mathrm{LiNbO}_{3}$, Appl. Phys. a 86 (2007) 165-170.

[29] L. Li, W. Nie, Z. Li, C. Romero, R.I. Rodriguez-Beltrán, J.R. Vázquez de Aldana, F. Chen, Laser-writing of ring-shaped waveguides in BGO crystal for telecommunication band, Optic Express 25 (2017) 24236-24241.

[30] T. Calmano, C. Kränkel, G. Huber, Laser oscillation in Yb:YAG waveguide beamsplitters with variable splitting ratio, Opt. Lett. 40 (2015) 1753-1756.

[31] Y. Liao, J. Xu, Y. Cheng, Z. Zhou, F. He, H. Sun, J. Song, X. Wang, Z. Xu, K. Sugioka, K. Midorikawa, Electro-optic integration of embedded electrodes and waveguides in
$\mathrm{LiNbO}_{3}$ using a femtosecond laser, Opt. Lett. (2008) 2281-2283.

[32] F. Laurell, T. Calmano, S. Müller, P. Zeil, C. Canalias, G. Huber, Laser-written waveguides in KTP for broadband Type II second harmonic generation, Optic Express 20 (2012) 22308-22313.

[33] N. Dong, F. Chen, J.R. Vazquez de Aldana, Efficient second harmonic generation by birefringent phase matching in femtosecond laser-inscribed KTP cladding waveguides, Phys. Status Solidi Rapid Res. Lett. 6 (2012) 306-308.

[34] Y. Jia, C. Cheng, J.R. Vázquez de Aldana, G.R. Castillo, B. del Rosal Rabes, Y. Tan, D. Jaque, F. Chen, Monolithic crystalline cladding microstructures for efficient light guiding and beam manipulation in passive and active regimes, Sci. Rep. 4 (2014) 5988.

[35] W. Nie, Y. Jia, J.R. Vazquez de Aldana, F. Chen, Efficient second harmonic generation in 3D nonlinear optical-lattice-like cladding waveguide splitters by femtosecond laser inscription, Sci. Rep. 6 (2016) 22310.

[36] S. Campbell, R.R. Thomson, D.P. Hand, A.K. Kar, D.T. Reid, C. Canalias, V. Pasiskevicius, F. Laurell, Frequency-doubling in femtosecond laser inscribed periodically-poled potassium titanyl phosphate waveguides, Optic Express 15 (2007) 17146-17150.

[37] S. Müller, T. Calmano, P.W. Metz, C. Kränkel, C. Canalias, C. Liljestrand, F. Laurell, G. Huber, Highly efficient continuous wave blue second-harmonic generation in fslaser written periodically poled Rb:KTiOPO 4 waveguides, Opt. Lett. 39 (5) (2014) 1274-1277.

[38] Rsoft Design Group, Computer software BeamPROP version 8.0, http://www. rsoftdesign.com..

[39] C. Chen, C.E. Rüter, M.F. Volk, C. Chen, Z. Shang, Q. Lu, S. Akhmadaliev, S. Zhou, F. Chen, D. Kip, Second harmonic generation of diamond-blade diced $\mathrm{KTiOPO}_{4}$ ridge waveguides, Optic Express 24 (2016) 16434-16439. 